UCRL-JC-128288

PREPRINT

\title{
Minimizing mapping-induced OPD errors when testing aspheric mirrors
}

\author{
R. M. Hudyma \\ G. Sommargren
}

This paper was prepared for and presented at the 23rd Annual International Symposium on Microlithography

Santa Clara, California

February 22-27, 1998

March 16, 1998

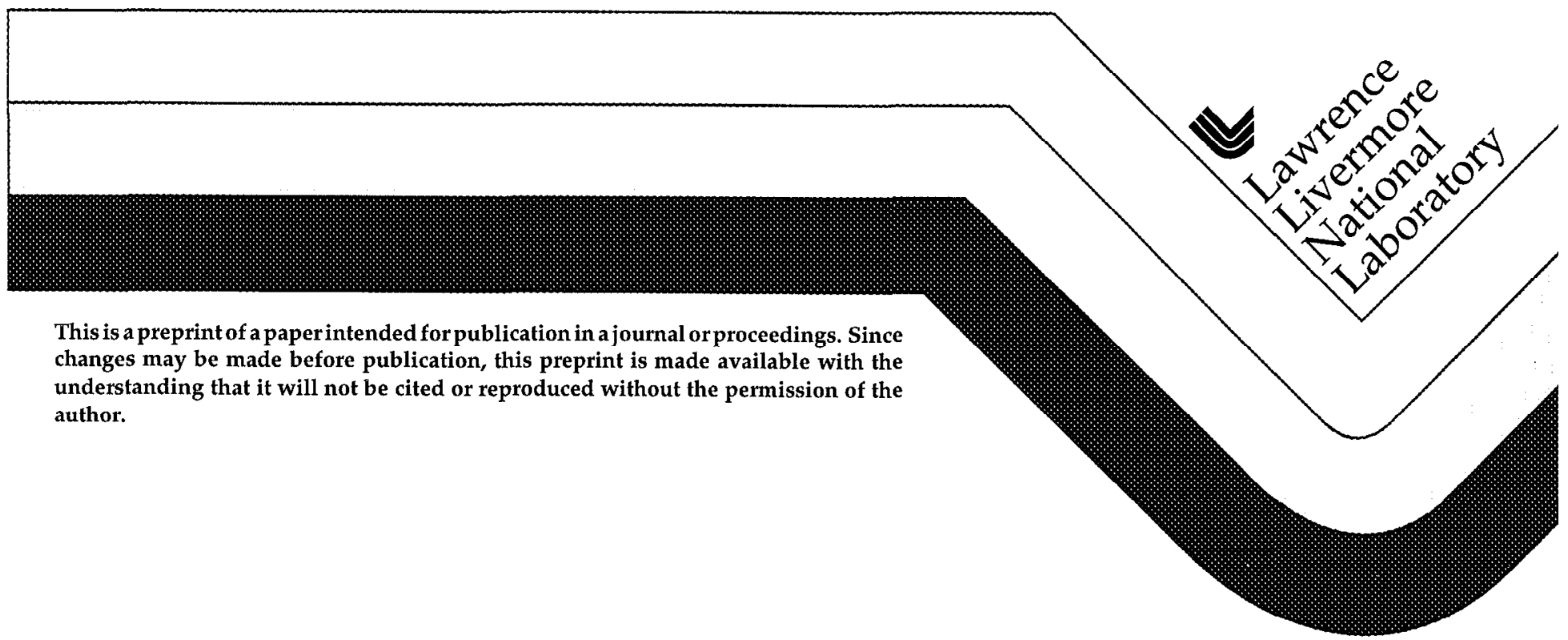




\section{DISCLAIMER}

This document was prepared as an account of work sponsored by an agency of the United States Government. Neither the United States Government nor the University of California nor any of their employees, makes any warranty, express or implied, or assumes any legal liability or responsibility for the accuracy, completeness, or usefulness of any information, apparatus, product, or process disclosed, or represents that its use would not infringe privately owned rights. Reference herein to any specific commercial product, process, or service by trade name, trademark, manufacturer, or otherwise, does not necessarily constitute or imply its endorsement, recommendation, or favoring by the United States Government or the University of California. The views and opinions of authors expressed herein do not necessarily state or reflect those of the United States Government or the University of California, and shall not be used for advertising or product endorsement purposes. 


\title{
Minimizing mapping-induced OPD errors when testing aspheric mirrors
}

\author{
Russell M. Hudyma and Gary Sommargren \\ Lawrence Livermore National Laboratory, L-395, Livermore, CA 94550
}

\begin{abstract}
Extreme ultraviolet (EUV) projection systems are designed with mild aspheres so that the mirrors can be tested at the center of curvature without null optics. The elimination of the null optics improves the fundamental accuracy of the test. However, this test configuration is not stigmatic, and the rays from the test wavefront and reference wavefront will not trace the same optical path through the viewing system. Effectively, the test and reference wavefront are sheared in the exit pupil of the viewing system. This shear leads to an OPD contribution from the viewing system that we label the mapping error. For visible light metrology, this induced OPD error can be a significant fraction of the EUV wavelength.
\end{abstract}

The origin of this OPD error is demonstrated using simple ray optics. Examples are presented illustrating the nature and magnitude of this error for off-axis aspheric sections. Methods for minimizing this OPD error are suggested.

Keywords: interferometric errors, aspheric surface measurement, precision interferometry

\section{ORIGIN OF MAPPING-INDUCED OPD ERRORS}

The viewing system of an interferometer is considered a "common path" optic that has no influence on the accuracy of the test. The argument is that rays in both the test and reference wavefront travel along the same paths through the viewing system. Since the rays travel along the same path, the accumulated OPL is the same and the viewing system contributes no additional OPD to the interferogram. In fact, the rays from both the test and reference wavefronts do not travel along the same optical path through the viewing system. This difference in "mapping" between the test and reference rays in the viewing system creates an additional OPD contribution that depends upon both the test surface and the design of the viewing system. This mapping-induced OPD error can be thought of as a shear of the test wavefront and reference wavefront in the exit pupil of the viewing system. We describe this OPD error source as simply the mapping error or shear error and use the terms interchangeably.

For typical applications, mapping error is on the order of several milliwaves at visible wavelengths and is safely ignored. However, this error contribution becomes significant for the high accuracy needed to test aspheric EUV projection optics. For example, a $0.005 \lambda$ error at $532 \mathrm{~nm}$ corresponds to an error of $2.66 \mathrm{~nm}$ or $0.2 \lambda$ at a wavelength of $13.1 \mathrm{~nm}$. This error alone is an order of magnitude larger than current surfacc figurc requircments. ${ }^{1}$

The origin of mapping error is easily understood by comparing the unfolded tested geometry for a spherical surface with that of an aspheric surface. A sphere tested at its center of curvature is illustrated schematically in Figure 1. For a given point on the mirror, 2 collinear rays leave the ideal point source that is placed at the center of curvature. The ray in the test wavefront accumulates optical path length $L_{1}$ from the point source to the mirror. The ray in the reference accumulates optical path length $\mathrm{L}_{3}$ from the point source to the camera. The incidence angle for the test ray at the surface is zero, so this ray reflects back upon itself and accumulates an optical path length $\mathrm{L} 2$ from the surface to the camera (notes that the reference and test rays overlap to the right of the point source). The test and reference rays meet at the camera and interfere. The lengths labeled $L_{1}, L_{2}$, and $L_{3}$ represent the optical path length along a given ray, not the physical path length. The viewing system is placed one focal length (f) away from the point source so that the reference wavefront is collimated at the camera plane. The camera plane is placed at a distance $f(1+f / R)$ from the viewing system so that the surface under test is imaged onto the camera. In the schematic, these distances are labeled relative to the first and second principal plane, respectively 


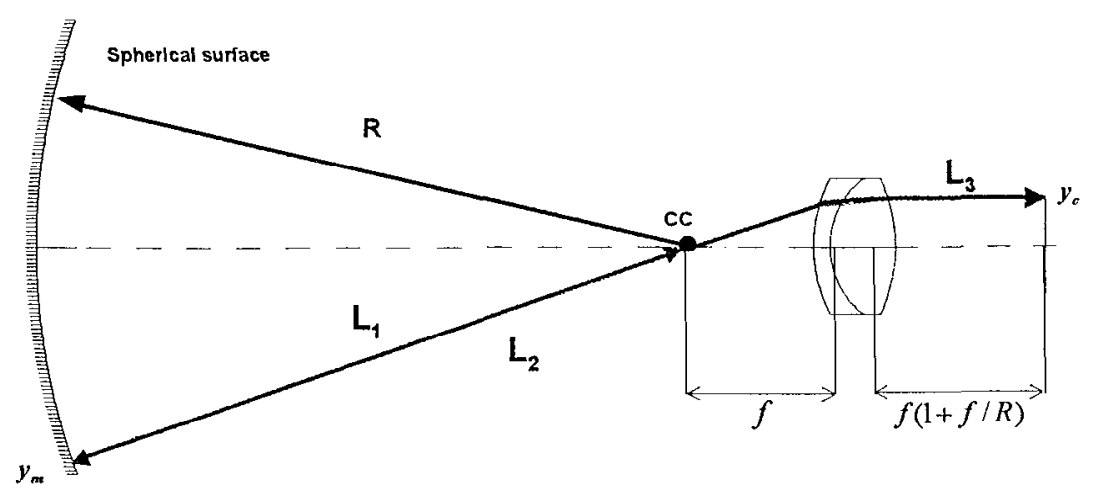

Figure 1. Spherical surface tested at its center of curvature. The imaging system (represented as a simple doublet) collimates the reference wavefront and images the surface under test so that point $y_{\mathrm{m}}$ and $\mathrm{y}_{\mathrm{c}}$ are conjugate

For point $y_{c}$ on the camera, the OPD between the test and reference rays is given by,

$$
O P D_{s}\left(y_{c}\right)=\left(L_{1}+L_{2}\right)-L_{3}+C
$$

where $C$ is a constant. From the figure, we see that the sum of OPL $L_{1}$ and $L_{3}$ is equal to $L_{2}$. The OPD on axis is equal to zero, so the constant $C$ is equal to $-2 R$. Substituting $L_{1}+L_{2}=L_{3}$ and $C=-2 R$ into (1), the $O P D$ between the test and reference ray with the point source at the center of curvature of a sphere is,

$$
O P D_{s}\left(y_{c}\right)=2\left(L_{1}-R\right)
$$

Equation (2) demonstrates the well-known fact that a center of curvature test is measures the surface figure error directly. For a perfect sphere tested at its center of curvature, the difference $\left(\mathrm{L}_{1}-\mathrm{R}\right)$ is identically zero for all points along the surface. Equation (2) is independent of $L_{2}$ and $L_{3}$, whose OPL holds contributions from the imaging system. In this case, the "common path" argument is valid and the viewing system has no impact on the measured OPD.

Figure 2 shows the raytrace diagram for an asphere tested at its center of curvature Again, collinear rays emerge from the ideal point source to define a test and reference wavefront. The ray representing a point propagating on the reference wavefront follows the same path as the reference ray in our spherical surface test shown in Figure 1. However, path $\mathrm{L}_{2}$ representing the reflected test ray follows a different path as it travels through the interferometer. This ray is no longer normally incidence at the mirror bccausc an aspheric surface has a curvature that constantly changes with radial position. The test ray will hit the entrance pupil of the viewing system (and emerge from the exit pupil) with a lateral shift with respect to the reference ray. This lateral shift in the pupil is labeled the shear $\mathrm{S}\left(\mathrm{y}_{\mathrm{m}}\right)$ in the figure. In effect, the points that interfere on the camera at $\mathrm{y}_{\mathrm{c}}$ are sheared in the exit pupil of the viewing system. This mapping or shear gives rise to an OPD error that depends on both the aspheric surface profile and the design viewing system.

The mapping error can be determined numerically by differencing two distinctly different phase maps on a point by point basis. First, the optical path difference for the test geometry must be evaluated at each point in the interferogram with no assumptions. For an arbitrary point $y_{c}$ on the camera, the difference in OPL between the test and reference rays for the aspheric test $\left(\mathrm{OPD}_{\mathrm{A}}\right)$ is given by,

$$
O P D_{A}\left(y_{c}\right)=\left(L_{1}+L_{2}\right)-L_{3}+C
$$

where $C$ is a constant. The axial OPD is again zero, setting $C$ equal to $-2 R_{v}$. However, $L_{2}$ no longer follows the $\mathrm{L}_{1} / \mathrm{L}_{3}$ path. This means that $\mathrm{L}_{1}+\mathrm{L}_{3} \neq \mathrm{L}_{2}$ (unless the imaging system is perfect). The expression for $\mathrm{OPD}_{\mathrm{A}}$ becomes,

$$
O P D_{A}\left(y_{c}\right)=\left(L_{1}+L_{2}\right)-L_{3}-2 R_{v}
$$


The points $y_{m}$ and $y_{c}$ are conjugate since the viewing system images the surface under test to the camera. If the viewing system had no aberrations, and the imagery would be perfect and all rays in the imaging bundle from $y_{m}$ to $y_{c}$ would have the same OPL. In this case, $L 1+L 3=L 2$. For this ideal imaging system, the optical path difference $\left(\mathrm{OPD}_{\mathrm{I}}\right)$ at point $\mathrm{y}_{\mathrm{c}}$ is,

$$
O P D_{I}\left(y_{c}\right)=2\left(L_{1}-R_{v}\right)
$$

The mapping error is the difference in $\mathrm{OPD}$ expressed by equation (4) and (5), i.e., $O P D_{m}=O P P D_{\mathrm{A}}-\mathrm{OPD}_{\mathrm{I}}$. Performing the subtraction we have,

$$
O P D_{m}\left(y_{c}\right)=L_{2}-\left(L_{1}+L_{3}\right)
$$

The mapping-induced OPD error leads to an apparent surface figure error that is one-half the OPD is computed via equation (6). To develop a map of this apparent surface figure error, the test geometry is raytraced and OPL $L_{1}, L_{2}$, and $\mathrm{L}_{3}$ are evaluated. An iterative raytrace routine must be used to find the test ray that intersects with the reference ray on the camera plane.

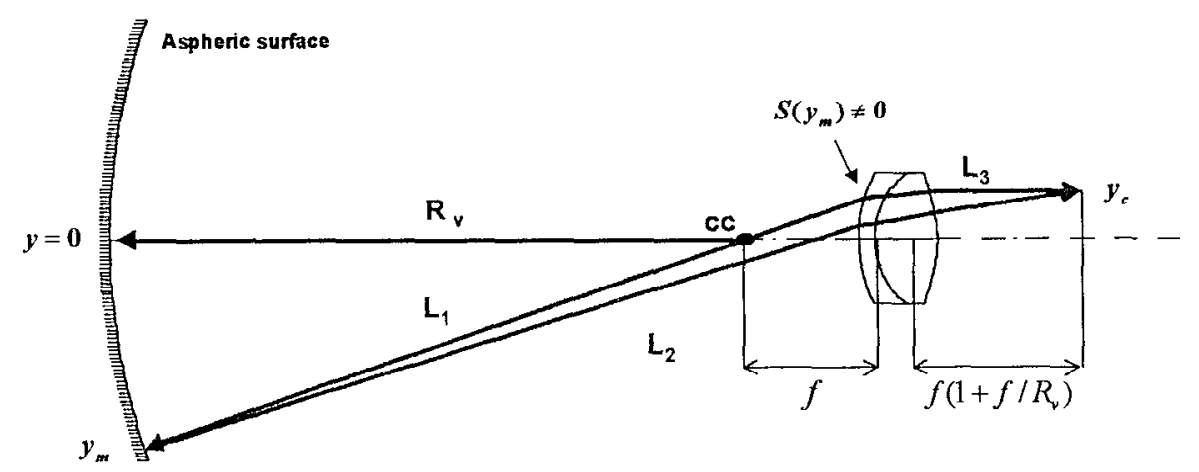

Figure 2. Aspheric surface tested at its center of curvature The reference and test wavefronts are separated as they enter the imaging system. Effectively, this separation is a shear of the reference and test wavefronts in the exit pupil of the viewing system. This shear causes and OPD error

\section{DESENSITIZING THE IMAGING SYSTEM TO MAPPING ERROR}

If the imaging system was perfect, the mapping error would be zero. However, it is exceedingly difficult to design and attempt to build a perfect imaging system. The design task is simplified by the realization that only 2 rays in the imaging bundle from $y_{m}$ to $y_{c}$, the ray that follows path $L 2$ and the ray that follows path $(L 1+L 3)$, need to have the same OPL. If those rays can identified and the shear quantified, then OPL targets can be placed directly on those rays so that the OPD between them can be driven to zero.

To develop the OPL constraints, the shear of the test and reference rays in the entrance pupil of the imaging system must first be examined. The shear characteristics depend upon the type of asphere being tested and can be represented in a vector field plot. The magnitude and orientation of the individual field vectors reveals the complexity of the design task. Figure 3 shows a field plot for a mild EUV asphere $(<3 \mu \mathrm{m}$ departure from the bestfit sphere across the off-axis section). This shear map reveals several general test characteristics. While layouts depicting rotationally symmetric aspheres were used to illustrate the origin of the mapping error, off-axis sections are tested in practice. The ring field projection geometry defines a test footprint that has the shape of a "kidney bean" across the off-axis section The viewing system is tilted by several degrees to center the section in the field of view of the imaging system. This mcans that the mapping crror will have bilateral, not rotational, symmetry as shown in the figure. Furthermore, a slight amount of defocus is added to the test to reduce the fringe density in the interference pattern on the CCD. This defocus creates a broad null region in the interferogram (and the shear map) where the mapping error is identically zero. This null region is a point of inflection in the test wavefront; the slope of the test wavefront changes sign through this null region. At the point of inflection, the mapping error is zero because the test ray reflects back upon itself. The direction of the shear changes sign with the changing slope of the test wavefront 
Figure 3. Vector field plot illustrating shear between test and reference wavefront as a function of mirror coordinates $\left(x_{m}, y_{m}\right)$ for an off-axis aspheric section. The shear (and the mapping error) has bilateral, but not rotational, symmetry. The aspheric section is not tested at its exact center of curvature defined by the vertex. A small amount of defocus is added to lower the fringe density across the off-axis section. This results in a null region in the interferogram where the mapping error is identically zero. The magnitude of the largest shear vector is approximately $012 \mathrm{~mm}$.

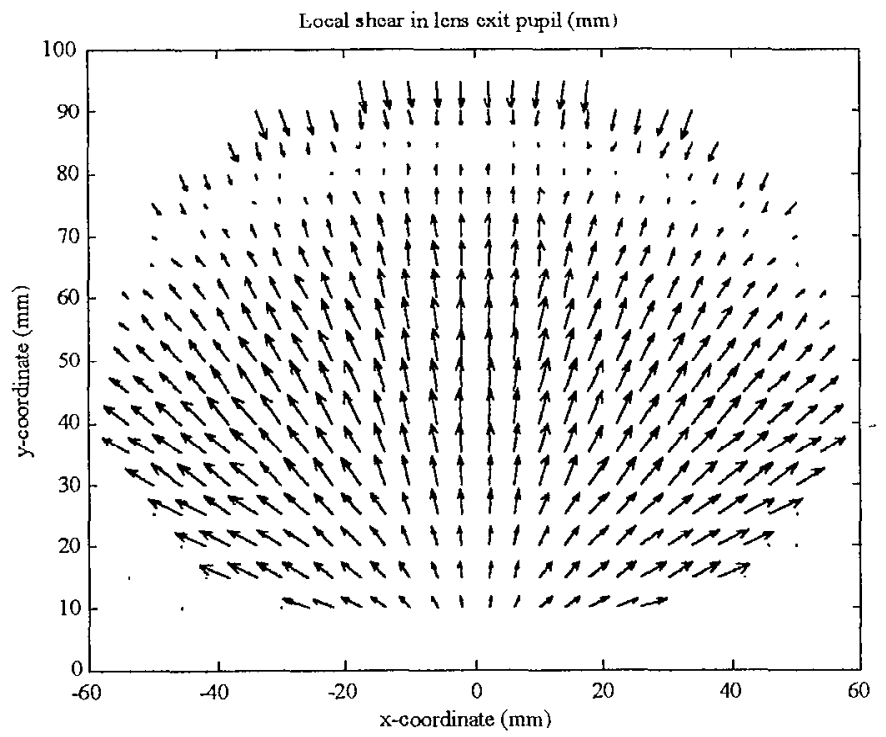

The imaging system of the interferometer is designed by minimizing the difference in OPL between the ray that traverses the $\left(\mathrm{L}_{1}+\mathrm{L}_{3}\right)$ path and the ray the follows the $\mathrm{L}_{2}$ path. This is accomplished by creating a user-defined merit function evaluated at several points in the field of view defined by the test geometry. This procedure desensitizes the imaging system to the shear error $\mathrm{S}\left(\mathrm{x}_{\mathrm{m}}, \mathrm{y}_{\mathrm{m}}\right)$. Although imaging systems for center of curvature tests can be designed using more traditional merit functions (RMS spot size, RMS wavefront error, etc.), we find that the creation of a merit function specifically tailored to minimize the mapping error yields more rapid convergence.

\section{REDUCTION OF MAPPING ERROR FOR A MILD ASPHERE}

The effectiveness of this approach is illustrated by example. The test geometry for a mild asphere was analyzed for mapping error assuming 2 different imaging systcm designs werc uscd. The apparent surface figure error induced by each system is compared graphically in Figure 4 . The error map on the left corresponds to an imaging system that was designed using a traditional merit function that minimized the RMS wavefront error across the entire imaging bundle. This optimization strategy led to a solution with excellent image quality, but very poor mapping error. This imaging system caused an apparent surface figure error that had a peak-to-valley (PTV) and root mean square (RMS) of $1.12 \mathrm{~nm}$ and $0.19 \mathrm{~nm}$, respectively
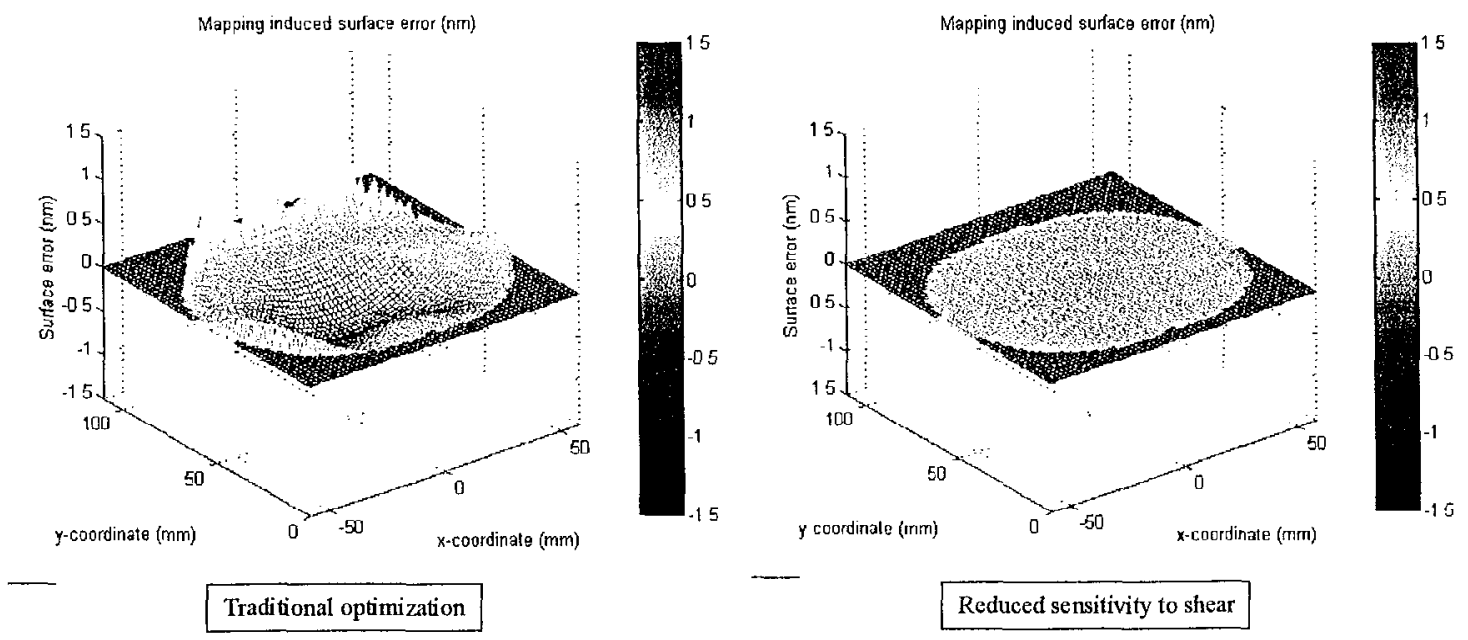

Figure 4 Mapping error for mild aspheres can be reduced to acceptable levels using proper design constraints. The plot scale is $\pm 1.5 \mathrm{~nm}$. 
The sensitivity to shear error can be reduced by targeting the change in OPL with shear to zero at several points across the surface under test. The procedure reduces the apparent surface figure error by a factor of $6 \mathrm{x}$ to $0.12 \mathrm{~nm}$ PTV and $0.03 \mathrm{~nm}$ RMS, respectively.

\section{STRATEGY TO REDUCE MAPPING ERROR FROM STRONG ASPHERE}

As mirror asphericity increases to $10-12 \mu \mathrm{m}$, the shear between the test and reference grows rapidly. The shear depends on many factors including the type of mirror and its vertex radius, and it is not uncommon for its magnitude to be greater than 2-3 mm for points near the edges of the test aperture. As this shear increases, it becomes more
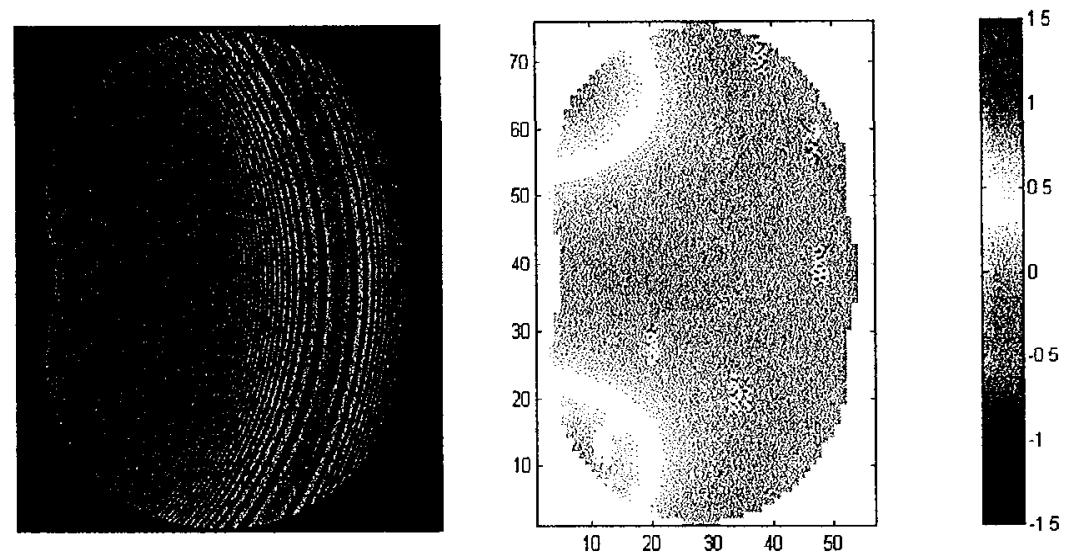

Figure 5a. Simulated interferogram and corresponding imaging lens error map scaled to represent surface figure (scale $\pm 1.5 \mathrm{~nm}$ ). The mapping error has been reduced using the merit function designed to minimize this error, but still shows poor statistics

difficult to drive this error to zero across the entire part without using a very complex imaging system. Figure 5 shows both a simulated interferogram and the corresponding error map for an aspheric section that has $10 \mu \mathrm{m}$ of departure from the best fit sphere across the off-axis section. The mapping error caused an apparent surface figure error of $0.19 \mathrm{~nm}$ RMS $(0.85 \mathrm{~nm}$ PTV). Clearly, these mapping error statistics are poor as we try to achieve a test accuracy of better than $0.25 \mathrm{~nm}$ RMS.

Figure 5a shows that the mapping error is zero in the null region of the interferogram. In this region, the test ray reflects back upon itself and the imaging lens has no impact on the measured OPD. This suggests an alternate strategy for minimizing mapping error: stitch together multiple subaperture data sets that use defocus to move null position across the test aperture. The idea of stitching multiple subaperture data sets together to test aspheres is not novel. This approach has been used primarily to circumvent the problem associated with sampling the high density fringe patterns typically associated with the aspheric testing. In the interferograms shown in Figurc 5a and 5b, the
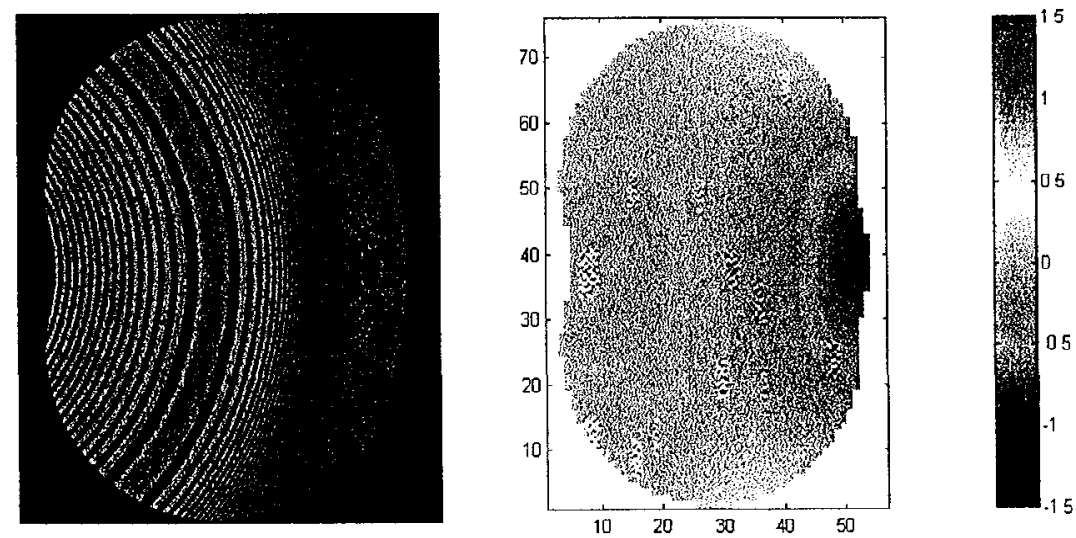

Figure $5 b$. Simulated interferogram and corresponding imaging lens error with new null location. Defocus is added to move the null fringe across the test aperture. Note that the mapping error is now effectively zero over the left half of test aperture.

minimum sampling density is 6 pixels per fringe, so stitching is not required to accommodate high fringe density However, stitching can be used to reduce the mapping error. Figure 5a shows that the mapping error is nearly zero 
across the right half of the test aperture, while Figure $5 \mathrm{~b}$ shows that it is nearly zero across the left half of the aperture By stitching these 2 data sets together, the mapping error can be nearly eliminated
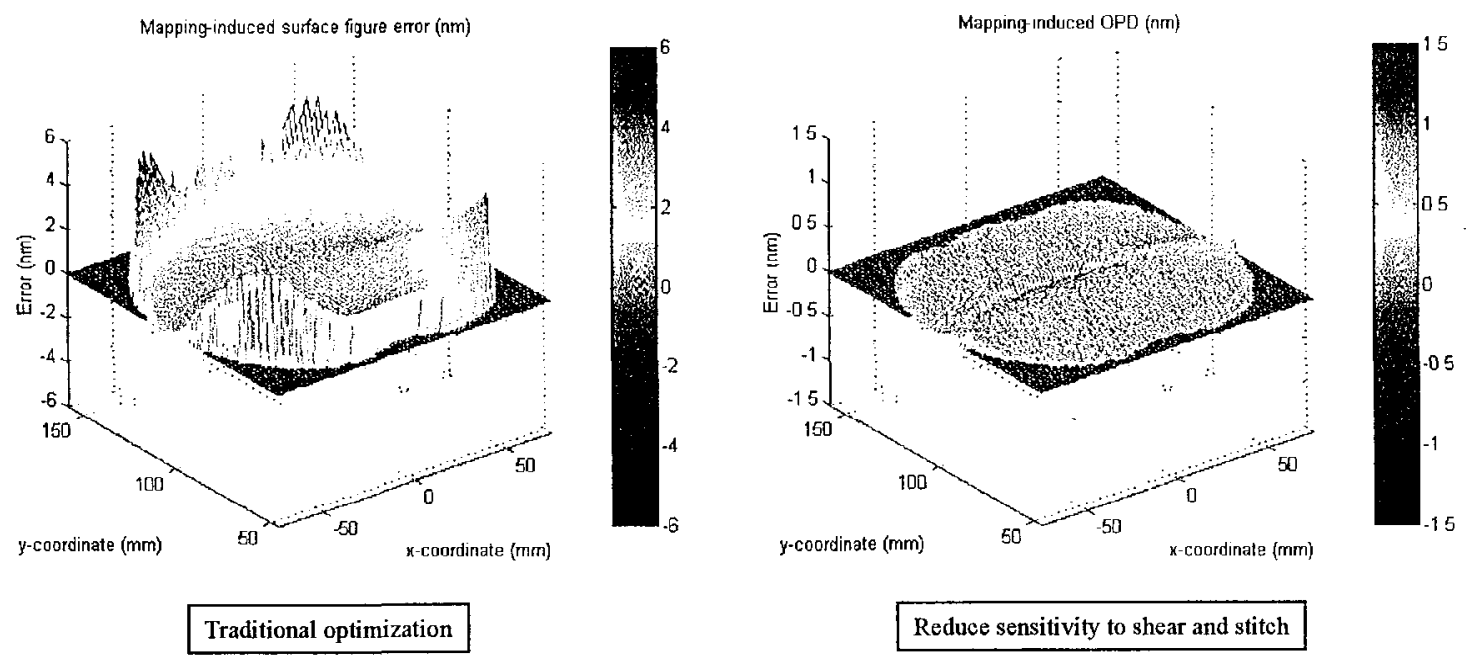

Figure 6. Comparison of apparent surface figure error for different 2 viewing systems designed to test a "strong" EUV asphere. Note that the scale for the error maps is $\pm 6 \mathrm{~nm}$ and $\pm 1.5 \mathrm{~nm}$. By using a combination of good design practice and stitching, the mapping error can be reduced by factor of $40 \mathrm{x}$.

Figure 6 was prepared to demonstrate the effectiveness of combining good design practice with stitching. Again we compare the apparent surface figure error from 2 different imaging systems. The first was designed by minimizing the RMS wavefront error across the entire imaging bundle. The second imaging system was designed specifically to minimize the mapping error. The imaging system that was designed using traditional metrics yields an apparent surface figure error of $1.10 \mathrm{~nm}$ RMS (5.6 nm PTV). This error alone is about $4 x$ larger than the surface figure accuracy that we are trying to achieve. Taking the optimal imaging system and stitching together 2 subaperture data sets to form a composite error map, we find that the apparent surface figure error caused by the imaging system has been reduced to $0.03 \mathrm{~nm}$ RMS (0.12 nm PTV). The combination of good design practice and stitching has reduced the RMS error from the imaging system by a factor of about $40 x$.

\section{CONCLUSION}

We have suggested a simple design technique for minimizing mapping-induced OPD errors from the imaging system when aspheric mirrors are tested at the center of curvature. For mild EUV aspheres $(<3 \mu \mathrm{m})$, good design practice alone is capable of reducing this systematic error to acceptable levels. For strong EUV aspheres (on the order of 10-12 $\mu \mathrm{m}$ ) good design practice and combined with subaperture stitching can be used to reduce the mapping error to levels that approach $0.025 \mathrm{~nm}$ RMS.

\section{ACKNOWLEDGEMENTS}

This work was performed under the auspices of the U.S. Department of Encrgy by the Lawrence Livermore National Laboratory under Contract No. W-7405-ENG-48. Funding was provided by the Extreme Ultraviolet Limited Liability Corporation under a Cooperative Research and Development Agreement.

\section{REFERENCES}

1. J. Taylor, et. al., "The fabrication and testing of optics for EUV projection lithography," Proc. SPIE Vol. 3331, 1998 (in press).

2. D. Sweeney, R. Hudyma, H. Chapman, and D. Shafer, "EUV Optical Design for a 100nm CD Imaging System," Proc. SPIE Vol. 3331, 1998 (in press). 


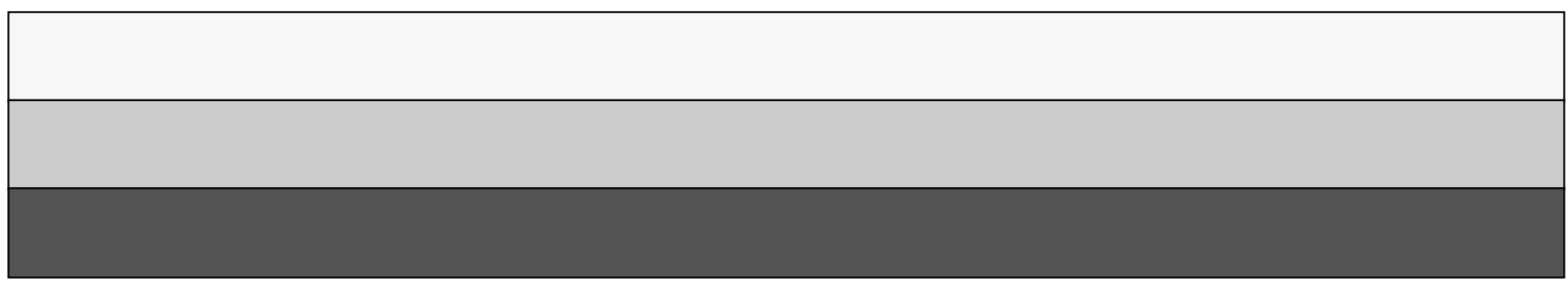

